\title{
RESEARCH HIGHLIGHT
}

\section{Prostate cancer biomarker: a key field to explore}

\author{
Jianqing Lin, Hushan Yang and William K Kelly \\ Asian Journal of Andrology (2013) 15, 358-359; doi:10.1038/aja.2013.1; published online 25 February 2013
}

$\mathbf{I}_{\mathrm{c}}^{\mathrm{n}}$ n order to achieve personalized cancer care, tools are needed to predict benefit from a specific therapy. Prostate cancer biomarker study is a key field to explore so that the natural course of a patient's disease can be predicted. A panel of inflammation/ immune-related and prostate cancer-related genes was screened by Ross et al. to assess genes predictive of castration-resistant prostate cancer (CRPC) survival. They found that a six-gene model was superior to traditionally used clinic-pathological variables. Although the clinic utility of this model remains to be determined, the blood based technical achievement and potential may eventually improve personalized cancer care.

CRPC is a late-stage prostate cancer typically evolved years after androgen deprivation therapy. Very recently, there has been a rapid increase in the number of effective systemic agents for men with metastatic CRPC (mCRPC), ${ }^{1}$ including abiraterone acetate and Enzalutamide (hormonal therapies), Sipuleucel-T (immunotherapy), Cabazitaxel (chemotherapy), Denosumab and Radium 223 (bone microenvironment targeting agents). However, the genetic heterogeneity of prostate cancer, the high cost of these therapies, and the uncertainty of the best use for these drugs in clinical decision-making, have highlighted the need for novel effective biomarkers to identify the right therapy and timing for the right patient and enable the monitoring of therapeutic response and disease progression.

Ross et al. ${ }^{2}$ recently published their findings in Lancet Oncology about the possibility of whole blood transcriptional profiling as prognostic biomarker for men with CRPC. Peripheral blood was prospectively collected from 62 men with CRPC on various treatment regimens who were enrolled in a training set and from 140 patients with CRPC in a

Department of Medical Oncology, Jefferson Kimmel Cancer Center, Thomas Jefferson University, Philadelphia, PA 19107, USA

Correspondence: Dr JQ Lin (Jianqing.lin@jefferson.edu) validation set from another institution. A panel of 168 inflammation/immune-related and prostate cancer-related genes was assessed with quantitative real-time PCR to assess genes predictive of survival. Latent class modeling was conducted to analyze the gene expression data, which generated a six-gene model (consisting of ABL2, SEMA4D, ITGAL, C1QA, TIMP1 and CDKN1A) that could separate CRPC patients into two risk groups: a low-risk group with a median survival of more than 34.9 months and a high-risk group with a median survival of 7.8 months $(P<0.0001)$. The prognostic utility of the six-gene model was validated in an independent cohort although less prominent than the training set (medium survival 18.5 months in low-risk group vs. 9.2 months in high risk-group, $P<0.0001$ ).

The prognostic ability of the six-gene model was compared with a model including only clinic-pathological variables that have been shown to be of prognostic significance in patients. ${ }^{3}$ The point-based nomograms have been developed based on clinical variables (typically include lactate dehydrogenase, prostate-specific antigen, alkaline phosphatase, Gleason sum, Eastern Cooperative Oncology Group performance status, hemoglobin and the presence of visceral disease). These nomograms have improved the ability to predict prognosis, but are not commonly used in busy clinical practice because of their complexity and limited predictive value to guide therapy. ${ }^{4}$ The author here concluded that the six-gene model resulted in a significantly higher area under the curve compared with a clinicopathological model (0.90 (95\% CI: 0.78-0.96) vs. 0.65 (95\% CI: $0.52-0.78)$; $P=0.0067)$. This is certainly interesting in that the six-gene model seems to be superior to these clinical variables which are mostly determined by disease but not the host.

Fresh biopsies would be preferable in cancer prognostication but obviously are difficult to obtain in mCRPC, given the particular propensity of mCRPC to metastasize to bone and induce blastic bone lesions. Patients are usually reluctant to have tissue biopsy if they have other options. Intratumor heterogeneity and the long natural history of prostate cancer limit the utility of archival tissues for biomarker studies to guide therapy for CRPC, a state when the genetic and epigenetic alterations accumulate in the metastatic deposits. Ideally, surrogate biomarkers, such as circulating tumor cells (CTCs) ${ }^{5}$ circulating tumor $\mathrm{DNA}^{6}$ and microRNA, ${ }^{7}$ may serve as a non-invasive 'liquid biopsy' to complement the use of archival tissues or bone biopsy. Increasing number of studies have shown that gene expression profiling of peripheral blood cells could yield diagnostic and prognostic information regarding various disease states. ${ }^{8-12}$ Whole blood offers several practical advantages in expression profiling studies compared to tumor tissue, including the minimally invasive nature of sample procurement, relative ease of standardization of sampling protocols, and the ability to conduct longitudinal sample evaluations.

The authors provided us an acceptable and reliable test (quantitative real-time PCR based) to start with. Nonetheless, the whole blood gene profiling may not reflect the genomics of metastatic tumor cells, because the vast majority of the RNAs are from peripheral blood mononuclear cells. Thus, the prognostic capacity of the six-gene signature may be further improved if CTCs are used for profiling. Moreover, although the initial 168 genes included a comprehensive panel of inflammation/immunityrelated and prostate cancer-related genes, it was still based on a hypothesis-driven candidate gene approach. A hypothesis-generating approach such as microarray or transcriptome sequencing that unbiasedly survey the complete gene expression spectrum may identify additional or different set of prognostic genes.

It is striking that all the six genes are related to host immune system which highlights the 
importance of host-tumor interactions in tumor development and progression. This finding suggests that the deregulated immune system dictates overall outcome for patients with CRPC. Using a similar approach, Olmos et al. ${ }^{13}$ reported a nine-gene signature which could stratify patients with CRPC into distinct prognostic groups. It is interesting that these genes are related to T-cell and B-cell functions. The explanation could be that gene expression profiles of peripheral blood mononuclear cells are somewhat related to tumor micro- and macro-environmental changes. ${ }^{14,15}$

These signatures provide us molecular snapshots of distinct host immune functions that may be relevant to patient survival. However, further investigation into the underlying biological mechanisms is needed. Future studies should clarify if any of these signature genes are functionally relevant, or are surrogate markers for other molecular processes. Although it is too early to conclude that whole-blood transcriptome profiling will be true game changers for the management of CRPC, at least this is an important promising step to move forward.

The next step would be the validation process. Large-scale prospective studies are needed to assess the practical usefulness of these signatures in the clinical context in which they may be used. The independent prognostic value of these signatures beyond those common clinic-pathological variables must also be explored in more depth. Furthermore, how the signatures perform compared with each other, and whether combination with other emerging biomarkers (e.g., CTC enumeration, proteins, radiographic biomarkers, etc.) will smooth personalized patient care, are to be determined. The authors suspected that these gene signatures might be the outcomes of interactions between tumor and host microenvironment. If this is true, several questions remain to be answered as to whether these signatures may be influenced by different timing of blood sampling and specific therapeutics, including immunotherapy patients received. Moreover, it is important to determine the potential utilization of these genes as predictive marker to monitor treatment response. To accelerate their clinical translation, it is extremely important to incorporate these novel candidate markers into prospective clinical trials. Decoding the interplay among multiple genetic, molecular, and host factors is challenging but exciting. It is possible that single blood test can guide us in clinic decision making. In addition, blood-based profiling of signaling transduction pathways may be another important aspect which will lead biomarkerdriven drug development and eventually facilitate personalized cancer therapy.

1 Chen Y, Scher HI. Prostate cancer in 2011: hitting old targets better and identifying new targets. Nat Rev Clin Oncol 2012; 9: 70-2.

2 Ross RW, Galsky MD, Scher HI, Magidson J, Wassmann $\mathrm{K}$ et al. A whole-blood RNA transcript-based prognostic model in men with castration-resistant prostate cancer: a prospective study. Lancet Oncol 2012; 13: 1105 13.

3 Halabi S, Small EJ, Kantoff PW, Kattan MW, Kaplan EB et al. Prognostic model for predicting survival in men with hormone-refractory metastatic prostate cancer. J Clin Oncol 2003; 21: 1232-7.

4 Shariat SF, Kattan MW, Vickers AJ, Karakiewicz PI, Scardino PT et al. Critical review of prostate cancer predictive tools. Future Oncol 2009; 5: 1555-84.

5 Danila DC, Fleisher M, Scher HI. Circulating tumor cells as biomarkers in prostate cancer. Clin Cancer Res 2011; 17: 3903-12.

6 Cortese R, Kwan A, Lalonde E, Bryzgunova O, Bondar $A$ et al. Epigenetic markers of prostate cancer in plasma circulating DNA. Hum Mol Genet 2012; 21: 3619-31.

7 Liu C, Kelnar K, Vlassov AV, Brown D, Wang J et al. Distinct microRNA expression profiles in prostate cancer stem/progenitor cells and tumor-suppressive functions of let-7. Cancer Res 2012; 72: 3393-404.

8 Alcorta D, Preston G, Munger W, Sullivan P, Yang JJ et al. Microarray studies of gene expression in circulating leukocytes in kidney diseases. Exp Nephrol 2002; 10: 139-49.

9 Bull TM, Coldren CD, Moore M, Sotto-Santiago SM, Pham DV et al. Gene microarray analysis of peripheral blood cells in pulmonary arterial hypertension. $\mathrm{Am} \mathrm{J}$ Respir Crit Care Med 2004; 170: 911-9.

10 Ma J, Liew CC Liew. Gene profiling identifies secreted protein transcripts from peripheral blood cells in coronary artery disease. J Mol Cell Cardiol. 2003; 35: 993-8.

11 Osman I, Bajorin DF, Sun TT, Zhong H, Douglas D et al. Novel blood biomarkers of human urinary bladder cancer. Clin Cancer Res 2006; 12: 3374-80.

12 Yang H, Qu F, Myers RE, Bao G, Hyslop T et al. Genetic variations in stem cell-related genes and colorectal cancer prognosis. J Gastrointest Cancer 2012; 43: 584-93.

13 Olmos D, Brewer D, Clark J, Danila DC, Parker C et al. Prognostic value of blood mRNA expression signatures in castration-resistant prostate cancer: a prospective, two-stage study. Lancet Oncol 2012; 13: 1114-24.

14 Toncheva D, Nacheva M. Chromosomal instability in cancer patients. Hum Genet 1998; 103: 367-71.

15 Dotan ZA, Dotan A, Ramon J, Avivi L. Altered mode of allelic replication accompanied by aneuploidy in peripheral blood lymphocytes of prostate cancer patients. Int J Cancer 2004; 111: 60-6. 